\title{
Strongly induced collapse in the Class 0 protostar NGC 1333 IRAS 4A
}

\author{
A. Belloche ${ }^{1}$, P. Hennebelle ${ }^{2}$, and P. André 3,4 \\ 1 Max-Planck-Institut für Radioastronomie, Auf dem Hügel 69, 53121 Bonn, Germany \\ e-mail: belloche@mpifr-bonn.mpg.de \\ 2 LERMA/LRA, École Normale Supérieure, 24 rue Lhomond, 75231 Paris Cedex 05, France \\ e-mail: patrick.hennebelle@ens. fr \\ ${ }^{3}$ Service d'Astrophysique, CEA/DSM/DAPNIA, C.E. Saclay, 91191 Gif-sur-Yvette Cedex, France \\ e-mail: pandre@cea.fr \\ 4 AIM, Unité Mixte de Recherche CEA-CNRS, Université Paris VII, UMR 7158, France
}

Received 23 December 2005 / Accepted 21 February 2006

\section{ABSTRACT}

\begin{abstract}
Context. The onset of gravitational collapse in cluster-forming clouds is still poorly known.
Aims. Our goal is to use the Class 0 protostar IRAS 4A, which is undergoing collapse in the active molecular cloud NGC 1333, to set constraints on this process. In particular we want to measure the mass infall rate and investigate whether the collapse could have been triggered by a strong external perturbation.

Methods. We analyze existing continuum observations to derive the density structure of the envelope, and use our new molecular line observations with the IRAM 30 m telescope to probe its velocity structure. We perform a detailed comparison of this set of data with a numerical model of collapse triggered by a fast external compression.

Results. Both the density and velocity structures of the envelope can be well fitted by this model of collapse induced by a fast external compression for a time of $1-2 \times 10^{4} \mathrm{yr}$ after the formation of the central protostar. We deduce a high mass infall rate of $0.7-2 \times 10^{-4} M_{\odot} \mathrm{yr}^{-1}$. The momentum required for the perturbation to produce this high mass infall rate is on the same order as the momenta measured for the NGC 1333 numerous outflows. Our analysis also shows that the turbulence is highly non uniform in the envelope, dropping from supersonic to subsonic values toward the center. The inner subsonic turbulence is most likely a relic of the conditions prevailing in the dense core before the onset of collapse.

Conclusions. The vigorous collapse undergone by IRAS 4A was triggered by a fast external compression, probably related to the expansion of a nearby cavity, which could have simultaneously triggered the collapse of the nearby Class 0 protostar IRAS 4B. This cavity could have been generated by an outflow but we have not found a good protostellar candidate yet.
\end{abstract}

Key words. stars: formation - stars: circumstellar matter - ISM: individual objects: NGC 1333 IRAS 4 -

ISM : kinematics and dynamics

\section{Introduction}

While the first phases of protostellar collapse in distributed starforming regions like the Taurus molecular cloud start to be observationally better constrained (e.g. Tafalla et al. 1998; Onishi et al. 1999; Motte \& André 2001; Belloche et al. 2002), little is known still about the onset of gravitational collapse in clusterforming clouds where this process is likely to be more violent (see André et al. 2004).

The protostar IRAS 4A is located in the active molecular cloud NGC 1333 that is forming low- and intermediate-mass stars in the Perseus complex (e.g. Sandell \& Knee 2001). The NGC 1333 region contains a double cluster of infrared sources, which is one of the least evolved embedded clusters known so far, with an age of $1-2 \times 10^{6} \mathrm{yr}$ (Lada et al. 1996; Lada \& Lada 2003). It also contains a population of younger stellar objects, including a few Class 0 protostars (e.g. Looney et al. 2000). IRAS 4A is one of these Class 0 protostars (André et al. 1993). It harbors a $1.8^{\prime \prime}$ binary system at a position angle PA of $-50^{\circ}$ (Reipurth et al. 2002) and is located in the vicinity of another young multiple system, IRAS 4B (Lay et al. 1995). Both sources are associated with molecular outflows oriented approximately in the north/south direction on a $10^{\prime \prime}$-scale (e.g. Choi 2001, 2005). On a larger scale, the IRAS $4 \mathrm{~A}$ outflow has a PA $\sim 45^{\circ}$ (e.g. Blake et al. 1995). The whole region is actually filled with about ten molecular outflows driven by young protostars that belong to the NGC 1333 protocluster (e.g. Knee \& Sandell 2000).

IRAS 4A was identified as a good infall candidate in the surveys of Mardones et al. (1997) and Gregersen et al. (1997). Di Francesco et al. (2001) used the IRAM Plateau de Bure interferometer to probe the inner parts of its envelope. They detected inverse P-Cygni profiles in $\mathrm{H}_{2} \mathrm{CO}\left(3_{12}-2_{11}\right)$ and $\mathrm{CS}(3-2)$, which they interpret as infall motions. They derived a high mass infall rate of $1.1 \times 10^{-4} M_{\odot} \mathrm{yr}^{-1}$, which is about 70 times higher than the standard accretion rate $\frac{c_{\mathrm{s}}^{3}}{G}$ at $10 \mathrm{~K}$ (Shu 1977). Such a high mass infall rate, if confirmed, cannot occur in an envelope collapsing spontaneously. The collapse of the IRAS 4A envelope was therefore probably triggered by a strong external perturbation. Several authors already argue that the numerous outflows have created cavities affecting the density structure of the molecular cloud and eventually triggered further star formation (Warin et al. 1996; Lefloch et al. 1998; Knee \& Sandell 2000; Quillen et al. 2005). In this respect, Sandell \& Knee (2001) propose that NGC 1333 is "an example of self-regulated star formation". 
However, it still remains to be shown that a collapse triggered by an external perturbation can match the density and velocity structure of a protostellar envelope such as IRAS 4A. This is the purpose of this work. The layout of the paper is as follows. Section 2 summarizes observational details. In Sect. 3 we derive the density structure of the envelope from a compilation of existing continuum data and interpret in terms of velocity structure our new molecular line observations done with the IRAM $30 \mathrm{~m}$ telescope. We compare these results in Sect. 4 to radiative transfer models of hydrodynamical simulations of collapse triggered by a fast increase in the external pressure (Hennebelle et al. 2003, 2004). Finally we discuss the implications in terms of triggered star formation in Sect. 5.

\section{Observations}

We carried out millimeter line observations with the IRAM $30 \mathrm{~m}$ telescope at Pico Veleta, Spain, in September and October 2001, and in August 2004, in the following molecular transitions: $\mathrm{HCO}^{+}(1-0), \mathrm{H}^{13} \mathrm{CO}^{+}(1-0), \mathrm{HC}^{18} \mathrm{O}^{+}(1-0), \mathrm{N}_{2} \mathrm{H}^{+}(1-0)$, $\mathrm{CS}(2-1), \mathrm{C}^{34} \mathrm{~S}(2-1), \mathrm{HCN}(1-0)$ at $3 \mathrm{~mm}, \mathrm{CS}(3-2), \mathrm{C}^{34} \mathrm{~S}(3-2)$, $\mathrm{N}_{2} \mathrm{D}^{+}(2-1)$ at $2 \mathrm{~mm}, \mathrm{H}_{2} \mathrm{CO}\left(3_{12}-2_{11}\right), \mathrm{H}_{2}^{13} \mathrm{CO}\left(3_{12}-2_{11}\right)$, $\mathrm{CS}(5-4)$, $\mathrm{N}_{2} \mathrm{D}^{+}(3-2)$ at $1.3 \mathrm{~mm}$, and $\mathrm{HCO}^{+}(3-2), \mathrm{H}^{13} \mathrm{CO}^{+}(3-2)$ at $1 \mathrm{~mm}$. The references to the frequencies we used are given in Sect. 3.2. The half-power beamwidths can be computed with the equation $H P B W\left({ }^{\prime \prime}\right)=\frac{2460}{v(\mathrm{GHz})}$. We used four SIS heterodyne receivers simultaneously and an autocorrelation spectrometer as backend. The spectral resolution was $20 \mathrm{kHz}$ at $3 \mathrm{~mm}$ and $2 \mathrm{~mm}$, and $40 \mathrm{kHz}$ at $1.3 \mathrm{~mm}$ and $1 \mathrm{~mm}$ in 2001 , and $10 \mathrm{kHz}$ at $3 \mathrm{~mm}$ and $20 \mathrm{kHz}$ at $1.3 \mathrm{~mm}$ in 2004 . The observations were done in single-sideband mode with sideband rejections of 0.01 at $3 \mathrm{~mm}$ and 0.05 at $2,1.3$, and $1 \mathrm{~mm}$. Accordingly, the calibration uncertainty was $\sim 10 \%$. The forward efficiencies $F_{\text {eff }}$ were 0.95 at $3 \mathrm{~mm}, 0.93$ at $2 \mathrm{~mm}, 0.91$ at $1.3 \mathrm{~mm}$, and 0.88 at $1 \mathrm{~mm}$. The main-beam efficiencies were computed using the Ruze function $B_{\text {eff }}=1.2 \epsilon \mathrm{e}^{-(4 \pi R \sigma / \lambda)^{2}}$, with $\epsilon=0.69, R \sigma=0.07$, and $\lambda$ the wavelength in $\mathrm{mm}$. The system temperatures ranged from $\sim 110 \mathrm{~K}$ to $\sim 160 \mathrm{~K}$ at $3 \mathrm{~mm}, \sim 190 \mathrm{~K}$ to $\sim 250 \mathrm{~K}$ at $2 \mathrm{~mm}, \sim 320 \mathrm{~K}$ to $\sim 670 \mathrm{~K}$ at $1.3 \mathrm{~mm}$, and $\sim 550 \mathrm{~K}$ to $\sim 1200 \mathrm{~K}$ at $1 \mathrm{~mm}$. The telescope pointing was checked every $\sim 2 \mathrm{~h}$ on Saturn, $3 \mathrm{C} 84$, and/or NRAO 140, and found to be accurate to $3^{\prime \prime}$ (rms). The telescope focus was optimized on Saturn and/or 3C 84 every $\sim 3 \mathrm{~h}$. Position-switching and on-the-fly observations were done with a reference position located at either (3000", 3000"), (1500", $\left.1500^{\prime \prime}\right),\left(0^{\prime \prime},-240^{\prime \prime}\right)$, or $\left(150^{\prime \prime}, 120^{\prime \prime}\right)$ relative to the envelope center $\alpha_{\mathrm{J} 2000}=03^{\mathrm{h}} 29^{\mathrm{m}} 10^{\mathrm{s}} 30, \delta_{\mathrm{J} 2000}=31^{\circ} 13^{\prime} 31.8^{\prime \prime}$, as measured in the $1.3 \mathrm{~mm}$ emission by Motte \& André (2001) ${ }^{1}$. The data were reduced with the CLASS software package (Buisson et al. 2002). The spectra were converted from antenna temperature to main-beam temperature using the equation $T_{\mathrm{mb}}=\frac{F_{\mathrm{eff}}}{B_{\mathrm{eff}}} T_{\mathrm{a}}^{*}$.

In addition, we downloaded from the JCMT archive $\mathrm{CS}(5-4), \mathrm{CS}(7-6), \mathrm{C}^{34} \mathrm{~S}(5-4), \mathrm{HCO}^{+}(4-3), \mathrm{H}^{13} \mathrm{CO}^{+}(3-2)$, and $\mathrm{DCO}^{+}(5-4)$ spectra observed toward IRAS 4A in February and July 1992, February 1994, November 1999, and October 2001. The data were converted into fits files with SPECX (Padman 1993; Matthews \& Jenness 1997), reduced with CLASS and converted to main-beam temperatures using beam efficiencies $\eta_{\mathrm{mb}}=0.69$ at $1 \mathrm{~mm}$ and 0.63 at $0.85 \mathrm{~mm}$ (see Maret et al. 2004, for the conversion to $T_{\mathrm{mb}}$ at JCMT).

\footnotetext{
${ }^{1}$ Note that our central position is offset from the two components observed by Reipurth et al. (2002) with VLA by $3^{\prime \prime}$ and $1.5^{\prime \prime}$ toward the West.
}

Table 1. Interferometric measurements of dust continuum emission.

\begin{tabular}{ccccccc}
\hline \hline Ref. $^{(1)} \lambda$ & $\begin{array}{c}H P B W \\
(\mathrm{~mm})\end{array}$ & $\begin{array}{c}\left.F_{\text {peak }}^{(2)} \times{ }^{\prime \prime}\right) \\
\left(\mathrm{mJy} \mathrm{beam}^{-1}\right)\end{array}$ & $\begin{array}{c}F_{\text {int }}^{(3)} \\
(\mathrm{mJy})\end{array}$ & $\begin{array}{c}\text { Size } \\
\left({ }^{\prime \prime} \times\right)\end{array}$ & Interf. \\
\hline D01 & 1.33 & $2.0 \times 1.7$ & $1200 \pm 16$ & $3100 \pm 470$ & $5.3 \times 4.1$ & PdBI \\
D01 & 2.04 & $3.2 \times 2.3$ & $450 \pm 14$ & $690 \pm 110$ & $4.4 \times 3.4$ & NMA \\
D01 & 2.19 & $3.4 \times 2.6$ & $420 \pm 10$ & $580 \pm 90$ & $4.2 \times 3.3$ & NMA \\
L00 & 2.7 & $5.5 \times 5.0$ & $351 \pm 3$ & $544 \pm 14$ & $25 \times 24$ & BIMA \\
& & $3.0 \times 2.8$ & $280 \pm 2$ & $526 \pm 9$ & $12 \times 18.5$ & BIMA \\
& & $1.2 \times 1.1$ & $172 \pm 2$ & $450 \pm 10$ & $5.4 \times 6.2$ & BIMA \\
D01 & 3.22 & $2.9 \times 2.6$ & $200 \pm 4.4$ & $320 \pm 49$ & $4.5 \times 3.7$ & PdBI \\
D01 & 6.92 & $2.1 \times 1.9$ & $16 \pm 0.29$ & $26 \pm 4$ & $3.3 \times 2.8$ & VLA \\
D01 & 13.3 & $3.8 \times 3.1$ & $3.1 \pm 0.27$ & $4.7 \pm 1.0$ & $6.3 \times 3.6$ & VLA \\
\hline
\end{tabular}

(1) References: L00: Looney et al. (2000); D01: Di Francesco et al. (2001). ${ }^{(2)}$ Quoted errors are statistical only and do not include any systematic calibration uncertainties. ${ }^{(3)}$ Flux integrated over a region of size listed in Col. 6.

Table 2. Single-dish measurements of dust continuum emission.

\begin{tabular}{lcccccl}
\hline \hline Ref. $^{(1)} \lambda$ & $H P B W$ & $\begin{array}{c}\mathrm{F}_{\text {peak }}^{(2)} \\
(\mathrm{mm})\end{array}$ & $\begin{array}{c}\mathrm{F}_{\text {int }}^{(3)} \\
\left({ }^{(3)}\right)\end{array}$ & $\begin{array}{c}\text { Size } \\
\left({ }^{\prime \prime} \times{ }^{\prime \prime}\right)\end{array}$ & Telescope \\
\hline S00 & 0.85 & 16.0 & $10.30 \pm 0.03$ & 12.9 & $30 \times 23$ & SCUBA \\
S01 & 0.85 & 14.0 & $\ldots$ & 9.05 & $14.2 \times 5.4$ & SCUBA \\
L98 & 1.25 & 11.0 & $3.10 \pm 0.01$ & 4.7 & $18.8 \times 18.8$ & $30 \mathrm{~m}$ \\
M01 & 1.25 & 11.5 & $4.10 \pm 0.02$ & 4.1 & $24 \times 24$ & $30 \mathrm{~m}$ \\
& & & & 7.3 & $97 \times 97$ & $30 \mathrm{~m}$ \\
\hline
\end{tabular}

(1) References: L98: Lefloch et al. (1998); S00: Smith et al. (2000); S01: Sandell \& Knee (2001); M01: Motte \& André (2001). ${ }^{(2)}$ Quoted errors are statistical only and do not include any systematic calibration uncertainties. ${ }^{(3)}$ Flux integrated over a region of size listed in Col. 6.

\section{Results}

\subsection{Continuum emission}

The mass distribution of the IRAS 4A envelope can be estimated from the dust continuum emission. From the literature we compiled the interferometric (Table 1) and single-dish (Table 2) measurements done so far. Masses were estimated by various authors using different assumptions for the dust emissivity, the dust temperature distribution, and/or the distance to NGC 1333. Here, we analyze this set of data homogeneously. We assume a distance of $318 \mathrm{pc}$, based on the Hipparcos parallactic measurements of the Perseus OB2 association (de Zeeuw et al. 1999), as discussed by Getman et al. (2002). With this distance, the bolometric luminosity derived by Sandell et al. (1991) becomes $11.6 L_{\odot}$. We use a dust emissivity $\kappa_{\lambda}=0.1 \times(\lambda / 250 \mu \mathrm{m})^{-\beta} \mathrm{cm}^{2} \mathrm{~g}^{-1}$ (Hildebrand 1983). The dust temperature profile is assumed to be fixed by the heating by the central protostar as suggested by Terebey et al. (1993):

$T_{\mathrm{d}}(r)=25\left(\frac{r}{6684 \mathrm{AU}}\right)^{-q}\left(\frac{L}{520 L_{\odot}}\right)^{q / 2}$

with $q=2 /(4+\beta)$ and $L=11.6 L_{\odot}$. We compute the mass within a spherical radius $R$ assuming optically thin emission and a temperature equal to $T_{\mathrm{d}}(R)$. However, this calculation overestimates the true mass because of the radius dependence of the temperature. When the temperature and the density are single power laws with slopes $-q$ and $-p$, respectively, and the Rayleigh-Jeans approximation is valid, the correction factor is $\left(1-\frac{q}{3-p}\right)$. For radii smaller than $\sim 9200 \mathrm{AU}, T_{\mathrm{d}}(r)>10 \mathrm{~K}$ and this approximation should be valid, provided the density profile follows a power 


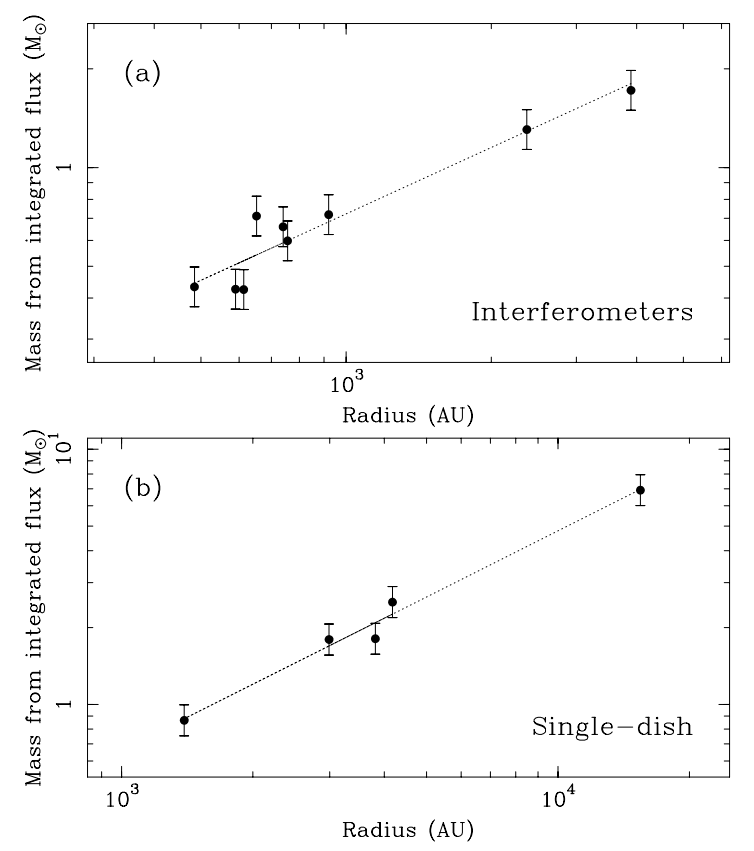

Fig. 1. Mass distribution estimated from the integrated flux of continuum emission measured with a) interferometers (see Table 1) and b) single-dish telescopes (see Table 2), and computed using the best-fit opacity exponent $\beta=0.85$.

law. For larger radii, we expect the dust grains to also be heated by the external UV radiation field via the photoelectric effect (e.g. Lesaffre et al. 2005). For simplicity, we assume that the dust temperature stays at $10 \mathrm{~K}$ for a radius larger than $9200 \mathrm{AU}$. In this case, the correction factor to compute the mass within a radius $R>9200 \mathrm{AU}$ is

$\left(\frac{q}{3-p-q} \times\left(\frac{R_{1}}{R}\right)^{3-p}+1\right)^{-1}$,

where $R_{1}(\sim 9200 \mathrm{AU})$ is the radius where the temperature starts to be constant.

Given these assumptions, the emissivity exponent minimizing the mass dispersion of the interferometric measurements shown in Fig. 1a is $\beta=0.85 \pm 0.15$, yielding $\kappa_{1.3 \mathrm{~mm}}=0.025 \pm$ $0.006 \mathrm{~cm}^{2} \mathrm{~g}^{-1}$. At larger radii with the single-dish measurements, we get $\beta=1.1 \pm 0.6$, which is poorly constrained because these measurements are not spread out enough in wavelength. In the following, we use $\beta=0.85$. The best power-law fits to the masses derived from the interferometric and singledish measurements are $M(r)=1.9 \times(r / 4200 \mathrm{AU})^{0.67} M_{\odot}$ and $M(r)=3.1 \times(r / 4200 \mathrm{AU})^{0.86} M_{\odot}$, respectively (see Fig. 1). A fit to all the data gives $M(r)=2.7 \times(r / 4200 \mathrm{AU})^{0.77} M_{\odot}$. For comparison, the mass of the singular isothermal sphere enclosed in $4200 \mathrm{AU}$ at $10 \mathrm{~K}$ is only $0.34 M_{\odot}$. We note that the interferometric measurements should resolve out any extended emission from the environment, and therefore give a more reliable estimate of the mass distribution within $13^{\prime \prime}$ in the IRAS 4A envelope.

Assuming a spherical geometry and a power-law density profile $n_{\mathrm{H}_{2}}=n_{0}\left(\frac{r}{r_{0}}\right)^{-p}$, the fit to the interferometric mass measurements yields $p=2.3$ and $n_{0}=2.1 \times 10^{5} \mathrm{~cm}^{-3}$ at $r_{0}=$ $4200 \mathrm{AU}$. By analyzing their $2.7 \mathrm{~mm}$ interferometric measurements, Looney et al. (2003) found no evidence of a circumstellar disk and they measured a power-law index $p$ between 1.7 and 2.3, which is consistent with our result (see their Table 1).
Note also that $n_{0}$ is 4 times higher than the density of a singular isothermal sphere at $10 \mathrm{~K}$, and this ratio is even higher at smaller radii.

\subsection{Spectroscopic signature of collapse}

In Fig. 2 we compare the $\mathrm{CS}, \mathrm{C}^{34} \mathrm{~S}, \mathrm{~N}_{2} \mathrm{H}^{+}, \mathrm{HCO}^{+}, \mathrm{H}^{13} \mathrm{CO}^{+}$, and $\mathrm{HC}^{18} \mathrm{O}^{+}$spectra that we obtained toward the central position of IRAS 4A with the IRAM $30 \mathrm{~m}$ telescope, as well as CS, $\mathrm{C}^{34} \mathrm{~S}, \mathrm{HCO}^{+}, \mathrm{H}^{13} \mathrm{CO}^{+}$, and $\mathrm{DCO}^{+}$spectra that we took from the JCMT archive. Among the latter, CS(5-4) and CS(7-6) were observed at a position that is slightly offset in right ascension (less than $\left.3^{\prime \prime}\right)$. We used the $\mathrm{CS}$ and $\mathrm{C}^{34} \mathrm{~S}$ frequencies measured by Gottlieb et al. (2003), the $\mathrm{N}_{2} \mathrm{H}^{+}(1-0)$ frequency determined by Dore et al. (2004), the $\mathrm{DCO}^{+}$frequencies measured by Caselli $\&$ Dore (2005), the $\mathrm{HCO}^{+}$frequencies from the CDMS database (as of October 2005, Müller et al. 2005), and the $\mathrm{H}^{13} \mathrm{CO}^{+}$and $\mathrm{HC}^{18} \mathrm{O}^{+}$frequencies determined by Schmid-Burgk et al. (2004).

The spectra of Fig. 2 show the classical signature of collapse, namely self-absorbed optically thick lines, with the blue peak stronger than the red one, and low-optical-depth spectra peaking in between (see Evans 1999; Myers et al. 2000). This signature is seen at $20^{\prime \prime}$ in the east/west direction in our $\operatorname{HCO}^{+}(3-2)$ map, but is only seen on the central position in our $\mathrm{CS}(5-4)$ map with a $10^{\prime \prime}$ spacing. Since the smoothing of our $\operatorname{CS}(5-4)$ map to the JCMT resolution yields a very similar spectrum to the JCMT one, the infall signature seen in the JCMT spectrum comes entirely from the inner $10^{\prime \prime}$ probed by the IRAM 30 m telescope.

In the classical spectroscopic signature of collapse motions (Evans 1999), the shift of the dip of the optically thick lines with respect to the systemic velocity of the source given by the centroid velocity of an optically thin line is an indication of the infall velocity of the absorbing material. A fit to the hyperfine multiplet of $\mathrm{N}_{2} \mathrm{H}^{+}(1-0)$ yields a low optical depth of $0.33 \pm 0.02$ for the isolated component 101-012, and a centroid velocity $V_{\mathrm{N}_{2} \mathrm{H}^{+}}=7.24 \pm 0.01 \mathrm{~km} \mathrm{~s}^{-1}$, which we take as the systemic velocity of the source. With respect to this systemic velocity, we measure shifts of $0.4,0.4$, and $0.5 \mathrm{~km} \mathrm{~s}^{-1}$ for $\mathrm{CS}(2-1)$, (3-2), and (5-4), respectively, and shifts of 0.5 and $0.5 \mathrm{~km} \mathrm{~s}^{-1}$ for $\mathrm{HCO}^{+}(3-2)$ and (4-3), respectively, all measured with an uncertainty of $0.1 \mathrm{~km} \mathrm{~s}^{-1}$. Di Francesco et al. (2001) measured a self-absorption at $7.64 \mathrm{~km} \mathrm{~s}^{-1}$ with the Plateau de Bure interferometer in $\mathrm{H}_{2} \mathrm{CO}\left(3_{12}-2_{11}\right)$, i.e. at the same velocity as the self-absorption in our $\mathrm{CS}(2-1)$ spectrum.

The $C^{34} S(2-1)$ and (3-2) spectra are also asymmetric, with even a marked dip for $\mathrm{C}^{34} \mathrm{~S}(2-1)$. These lines could be optically thick and self-absorbed, like the CS lines. On the other hand, they could be optically thin but probe two velocity components along the line of sight. This would be the case for an infalling envelope with strong depletion at the center (see the case of L1544 in Caselli et al. 2002), or with a small non-thermal velocity dispersion compared to the infall velocities, or for two independent velocity components, one being physically unrelated to the envelope. We address this question in Sects. 4 and 5. With a signal-to-noise ratio of 3, only one peak is detected in the JCMT $\mathrm{C}^{34} \mathrm{~S}(5-4)$ spectrum, at the same velocity as the $\mathrm{C}^{34} \mathrm{~S}(2-1)$ blue component.

\subsection{Extended wings tracing the outflow}

In Fig. 3 we present contour maps of the CS(2-1) and CS(5-4) line-wing emission overlaid on the intensity maps of the line core. Both show a clear bipolar morphology whose direction 

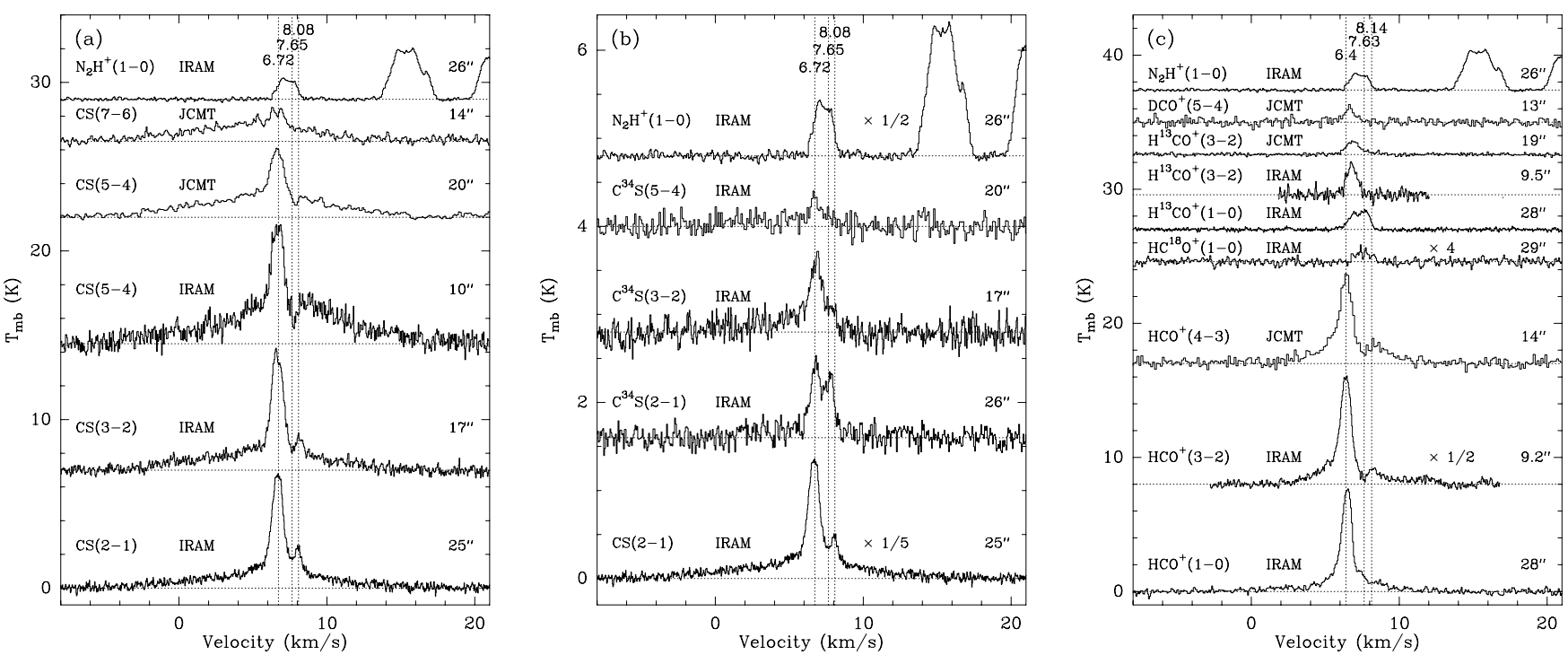

Fig. 2. Spectra toward the center of the IRAS 4A envelope obtained with the $30 \mathrm{~m}$ telescope or taken from the JCMT archive: a) CS and $\mathrm{N}_{2} \mathrm{H}^{+}$ spectra, b) $\mathrm{C}^{34} \mathrm{~S}$ spectra along with $\mathrm{CS}(2-1)$ and $\mathrm{N}_{2} \mathrm{H}^{+}(1-0)$, c) $\mathrm{HCO}^{+}, \mathrm{H}^{13} \mathrm{CO}^{+}, \mathrm{HC}^{18} \mathrm{O}^{+}$, and $\mathrm{DCO}^{+}$spectra along with $\mathrm{N}_{2} \mathrm{H}^{+}(1-0)$. The vertical dotted lines show the position of the blue peak, the red peak, and the dip of the CS(2-1) spectrum in a) and b), and the same for $\mathrm{HCO}^{+}(3-2)$ in $\left.\mathbf{c}\right)$. The temperature scale is in $T_{\mathrm{mb}}$. The beamwidth $(H P B W)$ is given for each spectrum.
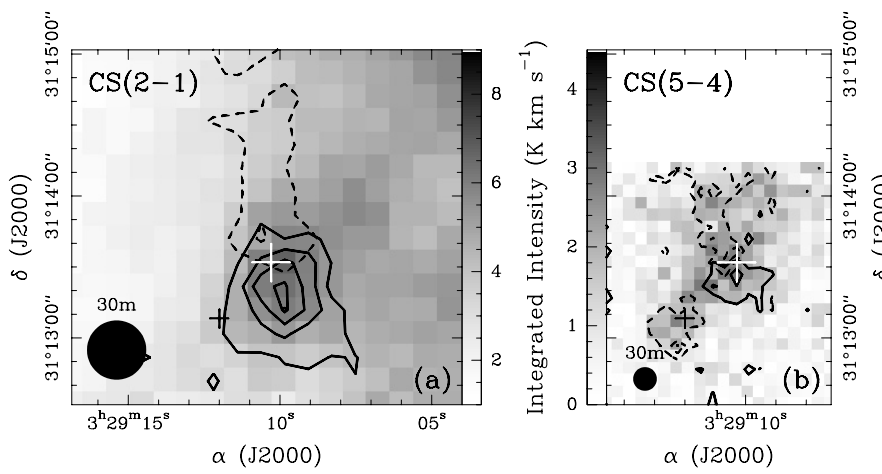

Fig. 3. a) $\mathrm{CS}(2-1)$ intensity maps toward IRAS $4 \mathrm{~A}$ integrated over $[-4.10,5.91] \mathrm{km} \mathrm{s}^{-1}$ (thick contours), $[6.06,8.31] \mathrm{km} \mathrm{s}^{-1}$ (grey scale), and $[8.46,15.50] \mathrm{km} \mathrm{s}^{-1}$ (dashed contours). The contours go from 1.5 to 6 by 1.5 and from 1.5 to 3 by $1.5 \mathrm{~K} \mathrm{~km} \mathrm{~s}^{-1}$, respectively. b) $\mathrm{CS}(5-4)$ intensity maps integrated over $[-1.37,5.91] \mathrm{km} \mathrm{s}^{-1}$ (thick contours), $[6.26,8.11] \mathrm{km} \mathrm{s}^{-1}$ (grey scale), and $[8.46,12.00] \mathrm{km} \mathrm{s}^{-1}$ (dashed contours). The contours are 2 and 4 , and 0.7 and $1.4 \mathrm{~K} \mathrm{~km} \mathrm{~s}^{-1}$, respectively. The intensity scale is $T_{\mathrm{a}}^{\star}$. The IRAM $30 \mathrm{~m}$ beam size is shown in each panel $(H P B W)$. The big/white and small/black crosses mark the positions of IRAS 4A and IRAS 4B, respectively.

$\mathrm{PA} \sim 10^{\circ}$ matches the direction of the outflow known in this source on a small scale (see Sect. 1). The wings of the CS (and $\mathrm{HCO}^{+}$) central spectra in Fig. 2 are thus certainly associated with the molecular outflow. They extend to higher velocities in CS (from $v=-2$ to $15 \mathrm{~km} \mathrm{~s}^{-1}$ ) than in $\mathrm{HCO}^{+}\left(3\right.$ to $11 \mathrm{~km} \mathrm{~s}^{-1}$ ).

\subsection{Maps of low optical depth tracers}

Figure 4 shows a map of $\mathrm{H}^{13} \mathrm{CO}^{+}(1-0)$ spectra taken toward IRAS 4A with the IRAM $30 \mathrm{~m}$ telescope. Nearly all spectra are double-peaked, as are our $\mathrm{C}^{34} \mathrm{~S}$ spectra with a slightly worse signal-to-noise ratio. The intensity of the blueshifted component peaks at the IRAS 4A central position, this behavior being more pronounced in the northeast/southwest direction than in the perpendicular direction. This component is thus certainly physically associated with the IRAS 4A envelope. By fitting two Gaussian

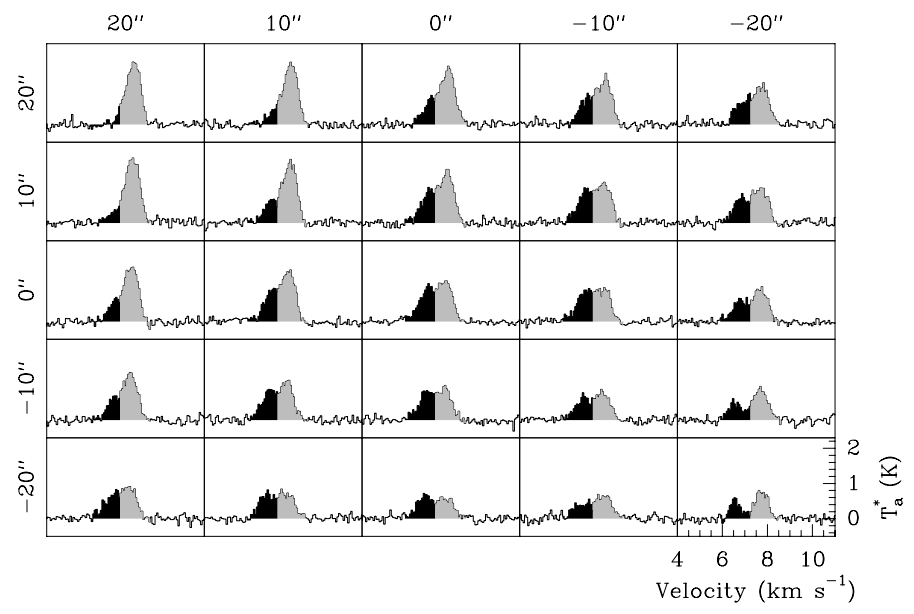

Fig. 4. Map of $\mathrm{H}^{13} \mathrm{CO}^{+}(1-0)$ spectra taken toward IRAS 4A with the IRAM $30 \mathrm{~m}$ telescope. Each spectrum is divided into two components, filled in black for $v<7.30 \mathrm{~km} \mathrm{~s}^{-1}$ and in grey for $v>7.30 \mathrm{~km} \mathrm{~s}^{-1}$. The half-power beam width is $28^{\prime \prime}$.

components to each spectrum, we measure for the blueshifted component a centroid velocity gradient of $\sim 9.7 \mathrm{~km} \mathrm{~s}^{-1} \mathrm{pc}^{-1}$ with a position angle PA $=38^{\circ}$ from North to East. On the other hand, the intensity of the redshifted component increases from the South-West to the North-East, and shows only a small velocity gradient of $2.4 \mathrm{~km} \mathrm{~s}^{-1} \mathrm{pc}^{-1}$ at $\mathrm{PA}=7^{\circ}$. The interpretation of these velocity gradients in terms of rotation is not straightforward.

In Fig. 5 we present large-scale maps of $\mathrm{N}_{2} \mathrm{H}^{+}(101-012)$ intensity integrated over the same velocity ranges as for $\mathrm{H}^{13} \mathrm{CO}^{+}(1-0)$ above (see Fig. 4). First of all, we notice that the $\mathrm{N}_{2} \mathrm{H}^{+}(101-012)$ emission has a local maximum very close to IRAS 4A in both Figs. 5a and b, which strongly suggests that both the blueshifted and redshifted components are related to the protostar. Second, these maps show that the $\mathrm{N}_{2} \mathrm{H}^{+}(101-012)$ emission traces the same structure as the dust continuum emission (e.g. Sandell \& Knee 2001; Hatchell et al. 2005): a filamentary structure extending along a direction with a position angle 

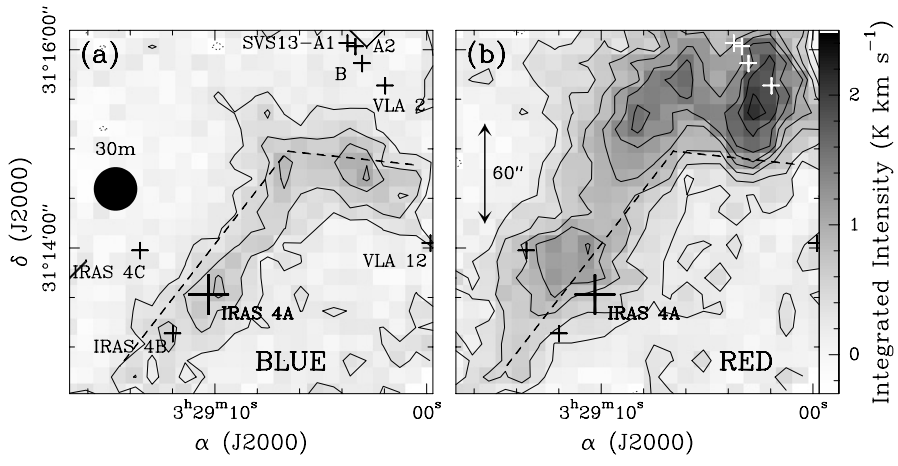

Fig. 5. Maps of $\mathrm{N}_{2} \mathrm{H}^{+}(101-012)$ intensity integrated over a) the blueshifted velocity range $[5.95,7.30] \mathrm{km} \mathrm{s}^{-1}$ and b) the redshifted velocity range $[7.30,8.65] \mathrm{km} \mathrm{s}^{-1}$, observed with the IRAM $30 \mathrm{~m}$ telescope (HPBW 26", shown on the left). The first contour and contour step are $0.25 \mathrm{~K} \mathrm{~km} \mathrm{~s}^{-1}$ on $T_{\mathrm{a}}^{\star}$ scale on both maps. The big cross marks the central position we used for IRAS 4A. The small crosses mark the positions of the VLA and BIMA continuum sources observed by Reipurth et al. (2002) and Looney et al. (2000). The dashed line separates the two components. It emphasizes the spatial shift between them and their shell-like structure.

$\mathrm{PA} \sim-45^{\circ}$, which bends at PA $\sim-90^{\circ}$ in the West. Furthermore, these maps provide additional kinematical information and show that the two components that we identified in $\mathrm{H}^{13} \mathrm{CO}^{+}(1-0)$ have the same morphology but are spatially shifted: the "blue" filament is shifted by $\sim 30^{\prime \prime}$ toward the South-West with respect to the "red" filament. Since they share the same morphology, they are likely to be physically related and not overlap by chance along the line of sight.

\section{Comparison to collapse models}

The classical signature of infall seen in our $\mathrm{CS}$ and $\mathrm{HCO}^{+}$observations (see Sect. 3.2 and Fig. 2) suggests that the envelope of IRAS 4A is collapsing, confirming the analysis of Di Francesco et al. (2001). They suggest that the inverse P-Cygni profiles that they observed with the IRAM Plateau de Bure Interferometer toward IRAS 4A in $\mathrm{H}_{2} \mathrm{CO}\left(3_{12}-2_{11}\right)$ are created by an infalling envelope with a mass infall rate of $1.1 \times 10^{-4} M_{\odot} \mathrm{yr}^{-1}$. This rate is nearly two orders of magnitude higher than the standard accretion rate $\frac{c_{\mathrm{s}}^{3}}{G}$ at $10 \mathrm{~K}$ (Shu 1977), and is the result of both higher densities and velocities (see Sects. 1 and 3.1). It is thus very unlikely that the collapse of this protostellar envelope has occurred spontaneously, so we investigate models of collapse induced by a fast external compression in this section.

\subsection{Model of collapse induced by compression}

Hennebelle et al. (2003, 2004) used a smoothed-particlehydrodynamics code to investigate the collapse of prestellar and protostellar cores driven from the outside by an increase in the external pressure. In the following, we compare our observations to their non-rotating model with rapid compression, i.e. $\phi=0.03$, where $\phi$ is the time-scale on which the external pressure doubles divided by the initial sound-crossing time. Such a rapid compression is required to get a density enhancement that matches the density profile of the IRAS 4A envelope (see Sect. 4.2). On the other hand, a more rapid compression produces infall velocities that are too high to get a good agreement with our observations toward IRAS 4A. The initial conditions of the model correspond to a core in stable hydrostatic equilibrium.
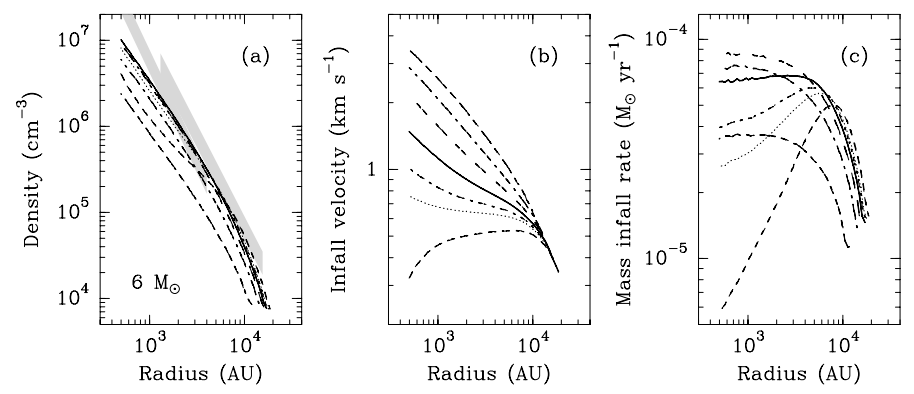

Fig. 6. a) Density, b) infall velocity, and c) mass infall rate profiles of the collapse model induced by compression described in Sect. 4.1 at times $0.8402,0.8533,0.8581,0.8657,0.8763,0.8920$, and $0.9249 \times 10^{6} \mathrm{yr}$, corresponding to renormalized times after the formation of the central protostar of approximately $-7,7,11,19,30,45$, and $78 \times 10^{3} \mathrm{yr}$, respectively. The thick line corresponds to $1.9 \times 10^{4} \mathrm{yr}$ and indicates the "best-fit" model used in Sect. 4.3 and shown in Fig. 7. The dashed areas in (a) show the constraints deduced for IRAS 4A from the interferometric and single-dish continuum measurements (see Sect. 3.1).

It is embedded in a hot rarefied external medium and has the structure of a Bonnor-Ebert sphere with a dimensionless radius $\xi_{\mathrm{b}}=3$, i.e. smaller than the dimensionless radius $\xi_{\mathrm{b}}=6.45$ of the critical Bonnor-Ebert sphere (e.g. Bonnor 1956). We normalized the model to a kinetic temperature of $10 \mathrm{~K}$ and a total mass of $6.0 M_{\odot}$.

\subsection{Mass distribution}

Figure 6a compares the evolution of the density profile of the model described in Sect. 4.1 with the density profiles deduced from the interferometric and single-dish continuum measurements toward IRAS 4A in Sect. 3.1. The fast external compression is responsible for a significant increase in the density profile compared to the profile expected for a singular isothermal sphere. The agreement with the profiles deduced from the observations is good in the range 1500-10000 AU at times $1-3 \times 10^{4} \mathrm{yr}$ after the formation of the central point mass, which are on the same order as the estimated lifetime of Class 0 protostars (André et al. 2000). The disagreement at smaller radii, the model being less dense than what we deduced from the observations assuming spherical symmetry, could be due to the presence of an unresolved disk not removed from the interferometric fluxes analyzed in Sect. 3.1. However, Looney et al. (2003) did not find any evidence of such a disk. At larger radii, the singledish measurements might be contaminated by large-scale emission from the cloud along the line of sight, which is not related to the protostellar envelope itself, although the dual-beam technique used for the observations should have removed most of it.

\subsection{Radiative transfer modeling}

We used the Monte-Carlo-based radiative transfer code MAPYSO in one spherical dimension (see Blinder 1997; Belloche et al. 2002) to compute the CS, $\mathrm{C}^{34} \mathrm{~S}$, and $\mathrm{N}_{2} \mathrm{H}^{+}$spectra that would be observed for a source set in collapse by fast compression, as described by the model of Sect. 4.1. In addition to the density and velocity profiles of the hydrodynamical model at time $1.9 \times 10^{4} \mathrm{yr}$ (see Figs. 6 and 7a, e), we used a uniform $\mathrm{CS} / \mathrm{C}^{34} \mathrm{~S}$ isotopic ratio of 22.5 but non-uniform abundance profiles (see Fig. 7c). For $\mathrm{N}_{2} \mathrm{H}^{+}$, we used the collision rates of $\mathrm{HCO}^{+}$with $\mathrm{H}_{2}$ (Flower 1999) and assumed a constant abundance of $4.1 \times 10^{-10}$. We did not model the hyperfine 


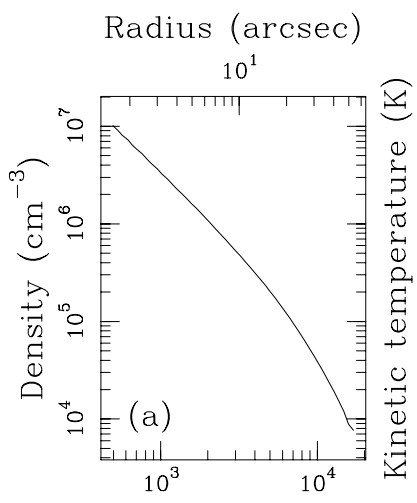

Radius (AU)
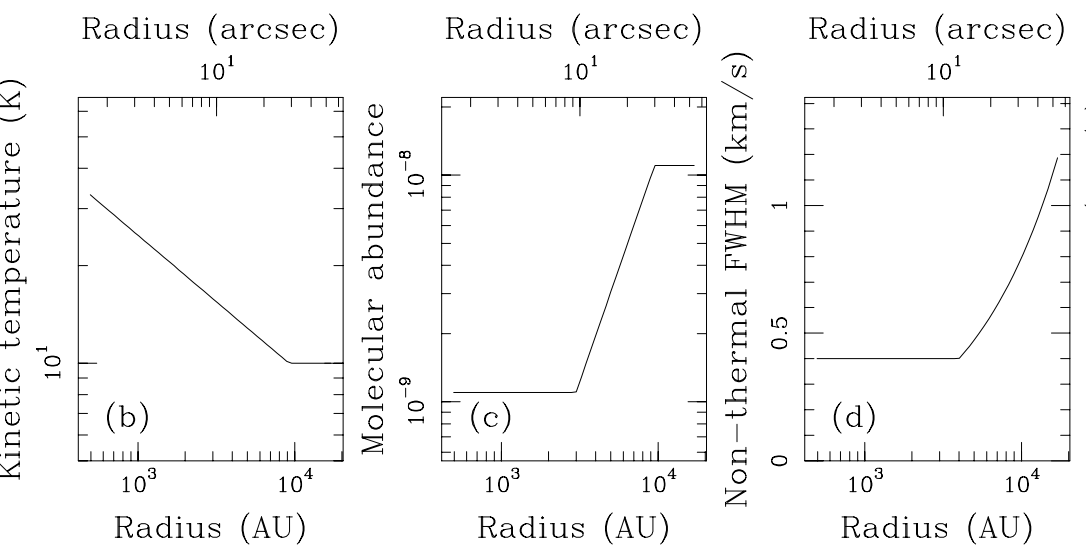

Radius (arcsec) $10^{1}$

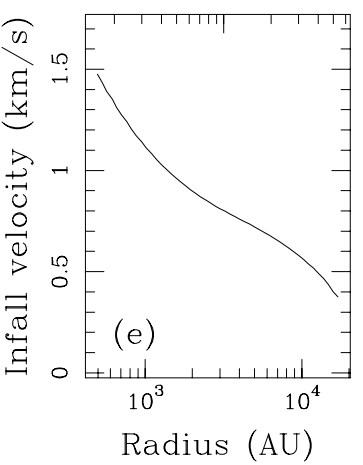

Fig. 7. a) Density, b) kinetic temperature, c) CS molecular abundance, d) non-thermal line broadening (FWHM), and e) infall velocity profiles of the collapse model at time $1.9 \times 10^{4}$ yr used for the radiative transfer modeling of Fig. 8 .

structure but simply rescaled the abundance effectively used for the calculations by the statistical weight ratio $\frac{3}{27}$ and fitted only the 101-012 optically thin component. For all molecules, we added a non-uniform non-thermal line broadening to the thermal broadening (see Fig. 7d). Although the hydrodynamical simulation was isothermal, we used a non-uniform kinetic temperature to compute the spectra, which are very sensitive to the heating by the central protostar. The temperature was set to the dust temperature profile described in Sect. 3.1 (see Fig. 7b).

In Fig. 8 we compare the synthetic spectra at time $1.9 \times$ $10^{4} \mathrm{yr}$ to the spectra observed toward IRAS $4 \mathrm{~A}$ along the longitude axis, i.e. roughly perpendicular to the outflow axis (see Fig. 3). The asymmetry and the absorption dips of the central CS(2-1) and CS(3-2) spectra are well reproduced. The asymmetry of these optically thick lines is enhanced toward the West and reduced toward the East at $\pm 20^{\prime \prime}$, which is not accounted for by our 1D spherical model. However, including some rotation could improve the modeled spectra in that respect (see Belloche et al. 2002, for such an effect in IRAM 04191+1522 - hereafter IRAM 04191). The line wings are not reproduced by our model, which was expected since they trace the ouflow that we did not include in our modeling (see Sect. 3.3). The relative contribution of the outflow gets stronger with the upper energy level of the transition, and even masks the red peak of the $\mathrm{CS}(5-4)$ spectrum. This spectrum is reasonably well-matched by the model, although the shape of the dip is not reproduced fully.

The central $C^{34} S(2-1)$ modeled spectrum matches the observed one well. It is optically thin $(\tau=0.4)$ and the two peaks result from the large infall velocities dominating the non-thermal line broadening in the inner parts of the envelope. We emphasize the importance of modeling the optically thin lines of a less abundant isotopologue simultaneously with the optically thick lines of the main molecule. This indeed reduces the space of free parameters drastically - especially the non-thermal line broadening and the abundance - and helps in deriving reliable infall velocities from the optically-thick, self-absorbed, asymmetric spectra. In particular, a first strong constraint is set on the abundance profile by the spatial variations of the $C^{34} S(2-1)$ integrated intensity, which imply a decrease in the abundance toward the center. Besides, a second constraint on the abundance in the external parts comes from the depth of the absorption dips of the $\mathrm{CS}(2-1)$ and $\mathrm{CS}(3-2)$ lines. As a result, this set of data reveals that CS is strongly affected by depletion in the IRAS 4A envelope (see Belloche et al. 2002, for a similar result in the Class 0 protostar IRAM 04191).

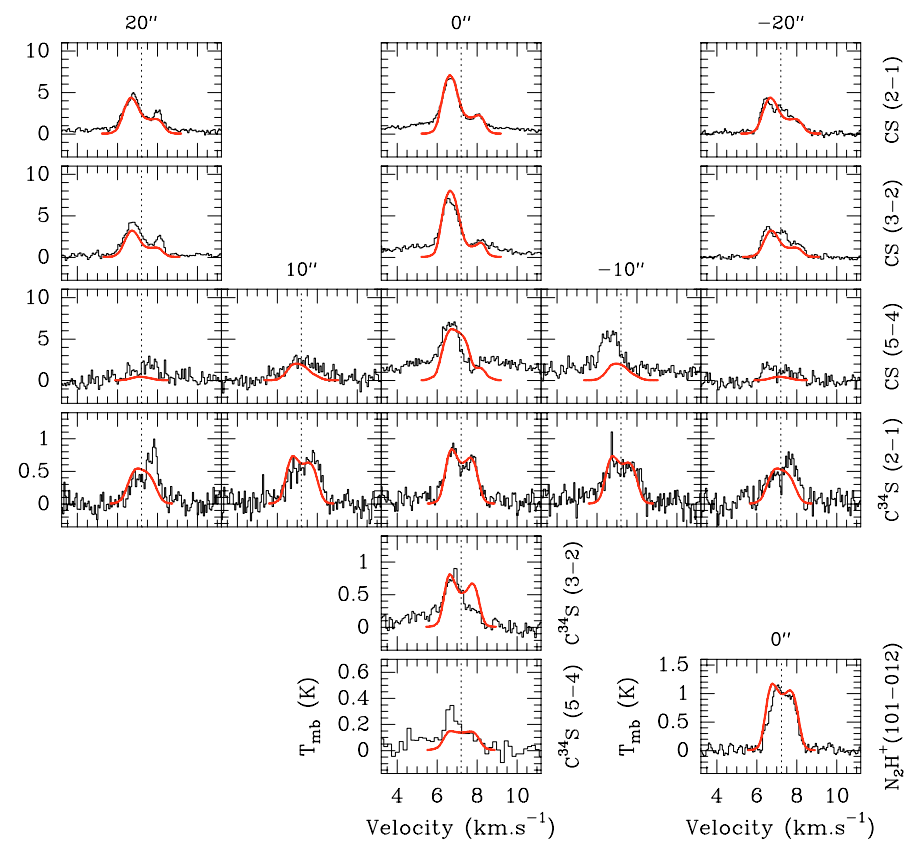

Fig. 8. $\mathrm{CS}, \mathrm{C}^{34} \mathrm{~S}$, and $\mathrm{N}_{2} \mathrm{H}^{+}$spectra (histograms) in units of main-beam temperature observed toward IRAS $4 \mathrm{~A}$ along the longitude axis (see references in Fig. 2). Synthetic spectra corresponding to the collapse model at time $1.9 \times 10^{4} \mathrm{yr}$ described in Sect. 4.1 are superimposed (red/thick line). The dotted line indicates the envelope systemic velocity used for the computation of the radiative transfer $\left(7.20 \mathrm{~km} \mathrm{~s}^{-1}\right.$ for CS and $\mathrm{C}^{34} \mathrm{~S}$, and $7.24 \mathrm{~km} \mathrm{~s}^{-1}$ for $\mathrm{N}_{2} \mathrm{H}^{+}$).

The $\mathrm{N}_{2} \mathrm{H}^{+}(101-012)$ line is also well reproduced, although the observed blue peak is not as blueshifted as in the model. The opacity of the modeled spectrum is 0.24 . It agrees reasonably well with the opacity obtained from the Gaussian fit to the hyperfine structure (see Sect. 3.2), and this small discrepancy may be due to our simplified treatment of the radiative transfer of $\mathrm{N}_{2} \mathrm{H}^{+}$. With the uniform abundance, the $\mathrm{N}_{2} \mathrm{H}^{+}(1-0)$ transition is more sensitive to the inner region than the $\mathrm{C}^{34} \mathrm{~S}(2-1)$ line. We tried a model that included depletion of $\mathrm{N}_{2} \mathrm{H}^{+}$above $\sim 10^{6} \mathrm{~cm}^{-3}$ (see Belloche \& André 2004, for $\mathrm{N}_{2} \mathrm{H}^{+}$depletion in IRAM 04191), but the shape of the line was worse, the blue and red peaks being much more pronounced than they are in the observed spectrum. The reason the uniform abundance produces a profile with less pronounced blue and red peaks is that, in this case, the beam picks up more material at low velocities projected along the line 
of sight, i.e. the material infalling along a direction close to the plane of the sky. This shows that the double-peaked profile of the $\mathrm{C}^{34} \mathrm{~S}(2-1)$ line is due not only to the large infall velocities compared to the non-thermal broadening, as stated above, but also to the depletion in the central region. In our model, the shape of the $\mathrm{N}_{2} \mathrm{H}^{+}(101-012)$ line therefore indicates no depletion of $\mathrm{N}_{2} \mathrm{H}^{+}$ (or very little) in the inner regions.

As noted above, the non-thermal line broadening in the inner parts of the envelope is strongly constrained to be small $\left(\Delta V_{\mathrm{NT}} \sim 0.4 \mathrm{~km} \mathrm{~s}^{-1}\right.$, i.e. $\left.\sigma_{\mathrm{NT}} \sim 0.17 \mathrm{~km} \mathrm{~s}^{-1}\right)$ by the doublepeaked line shape of the low-optical-depth $\mathrm{C}^{34} \mathrm{~S}(2-1)$ spectrum. The model with $\Delta V_{\mathrm{NT}}=1.1 \mathrm{~km} \mathrm{~s}^{-1}$ in Fig. 9 indeed shows that a larger broadening smoothes out the $\mathrm{C}^{34} \mathrm{~S}(2-1)$ double-peaked line profile which traces the infall velocities of the front and rear hemispheres. It also broadens the $\mathrm{N}_{2} \mathrm{H}^{+}(101-012)$ spectrum too much and produces wings that have not been observed. On the other hand, the $\mathrm{CS}(2-1)$ and (3-2) broad absorption dips require a much larger non-thermal line broadening in the external parts of the envelope where they are produced $\left(\Delta V_{\mathrm{NT}} \sim 1.2 \mathrm{~km} \mathrm{~s}^{-1}\right.$, i.e. $\left.\sigma_{\mathrm{NT}} \sim 0.5 \mathrm{~km} \mathrm{~s}^{-1}\right)$. The model with $\Delta V_{\mathrm{NT}}=0.4 \mathrm{~km} \mathrm{~s}^{-1}$ in Fig. 9 indeed shows that a smaller broadening cannot produce the broad absorption dips observed in $\mathrm{CS}(2-1)$ and $\mathrm{CS}(3-2)$. We have, therefore, strong evidence that the non-thermal line broadening is not uniform in the IRAS 4A envelope, decreasing by nearly a factor of three toward the center, from supersonic down to subsonic values. This conclusion agrees quite well with the small linewidths $\Delta V_{\mathrm{NT}} \sim 0.5 \mathrm{~km} \mathrm{~s}^{-1}$ observed toward IRAS 4A in $\mathrm{N}_{2} \mathrm{H}^{+}(1-0)$ by Di Francesco et al. (2001) on small scales with $\mathrm{PdBI}(r \sim 1000 \mathrm{AU})$ and the much larger linewidths $\Delta V_{\mathrm{NT}} \sim 1.0-1.9 \mathrm{~km} \mathrm{~s}^{-1}$ measured in the NGC 1333 molecular cloud with the low-density tracer $\mathrm{C}^{18} \mathrm{O}(1-0)$ by Warin et al. (1996).

The time elapsed since the point-mass formation in the collapse model is well constrained by the peak separations and the linewidths of the CS and $C^{34} \mathrm{~S}$ spectra. The infall velocities of the model at time $7 \times 10^{3} \mathrm{yr}$ in Fig. 10 are not high enough to produce the right separation of the peaks of the $\mathrm{CS}(3-2)$ and $\mathrm{C}^{34} \mathrm{~S}(2-1)$ lines. On the other hand, the much higher infall velocities of the model at time $4.5 \times 10^{4} \mathrm{yr}$ produce spectra that are too broad. As a result, we find a good match for a time of $1-2 \times 10^{4}$ yr elapsed since the formation of the central protostar.

Finally, we could not find a better match in the framework of our 1D spherical modeling for the $C^{34} S(3-2)$ and $C^{34} S(5-4)$ central spectra. The modeled spectra of Fig. 8 are not asymmetric enough for these two lines. Since our modeling suggests low optical depths of 0.35 and 0.04 for these two lines, it is very unlikely that the observed asymmetry results from an optical depth effect. This instead suggests that the material emitting in these two lines at the blue and redshifted velocities - i.e. in the rear and front hemispheres, respectively, in the framework of this collapse model - is denser for the former and less dense for the latter than assumed in the model.

\section{Discussion}

\subsection{A vigorous collapse induced by a fast compression}

In Sect. 4 we found very good agreement between the continuum and $\mathrm{CS} / \mathrm{C}^{34} \mathrm{~S} / \mathrm{N}_{2} \mathrm{H}^{+}$line observations toward IRAS $4 \mathrm{~A}$ and the collapse model of Hennebelle et al. (2003) induced by a fast external compression with $\phi=0.03$. The best match is obtained for a time of $1-2 \times 10^{4}$ yr elapsed since the formation of the central protostar. This corresponds to a mass infall rate of $4-7 \times 10^{-5} M_{\odot} \mathrm{yr}^{-1}$ in the range of radii $\left[10^{3}, 10^{4}\right] \mathrm{AU}$ (see

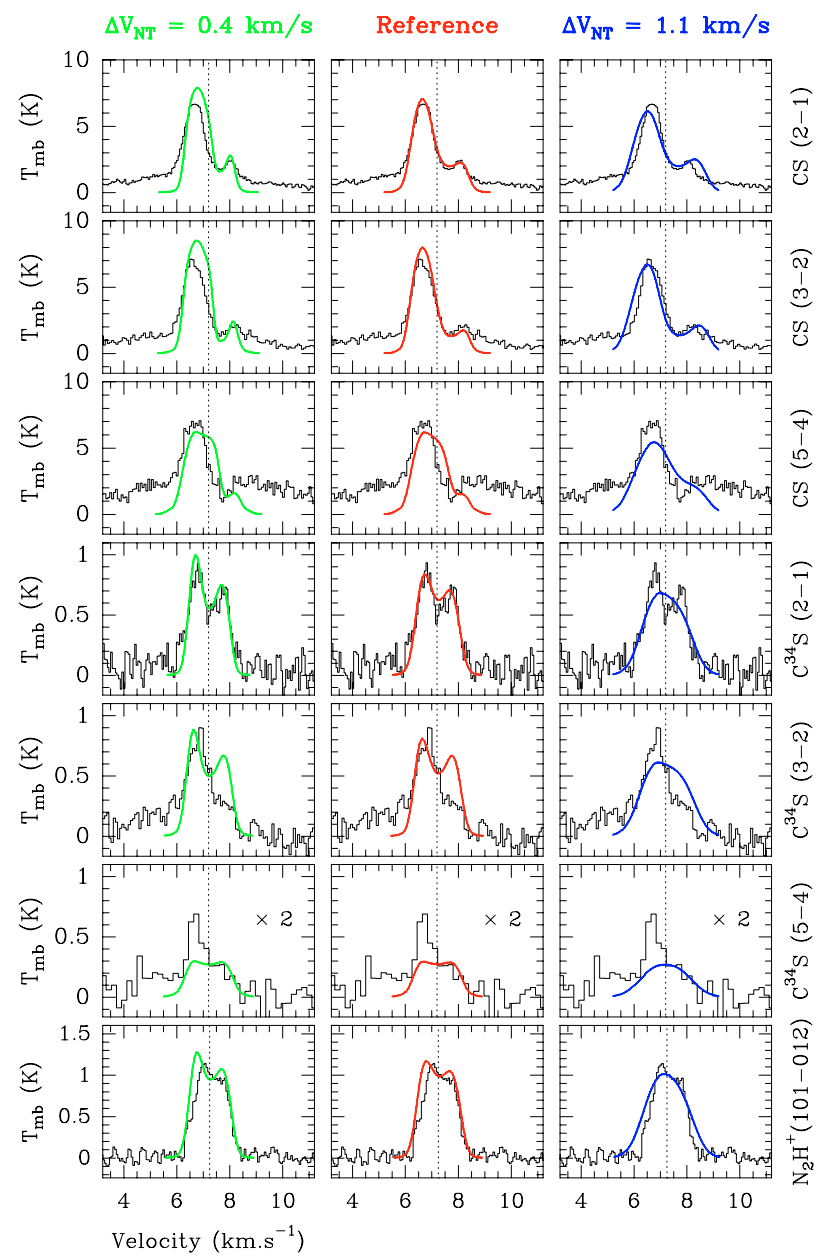

Fig. 9. $\mathrm{CS}, \mathrm{C}^{34} \mathrm{~S}$, and $\mathrm{N}_{2} \mathrm{H}^{+}$spectra in units of main-beam temperature observed toward the center of IRAS 4A (histograms). Each column shows a different model (thick line). Synthetic spectra corresponding to the collapse model at time $1.9 \times 10^{4} \mathrm{yr}$ described in Sect. 4.1 are superimposed in the central column (in red). In the left and right columns, the synthetic spectra (in green and blue) were obtained with the same input model, except for the non-thermal broadening, which was in both cases uniform with $\Delta V_{\mathrm{NT}}=0.4$ and $1.1 \mathrm{~km} \mathrm{~s}^{-1}$, respectively. The dotted line indicates the systemic velocity of the envelope that was used to compute the radiative transfer (see Fig. 8).

Fig. 6c). However, the actual mass infall rate is probably even higher since the density profile of our model does not perfectly match (and underestimates it by about 25\%) the density profile deduced from the observations (see Fig. 6a). Also, the dust opacity we used in Sect. 3.1 is 2.5 times larger than the opacity usually assumed for protostellar envelopes (e.g. Motte \& André 2001). Therefore we estimate that the actual mass infall rate is most probably in the range $0.7-2 \times 10^{-4} M_{\odot} \mathrm{yr}^{-1}$. This is in very good agreement with the mass infall rate of $1.1 \times 10^{-4} M_{\odot} \mathrm{yr}^{-1}$ deduced by Di Francesco et al. (2001) from their PdBI observations. As already mentioned in Sect. 4, such a mass infall rate of $1 \times 10^{-4} M_{\odot} \mathrm{yr}^{-1}$ is 60 times higher than the standard accretion rate $\frac{c_{\mathrm{s}}^{3}}{G}$ at $10 \mathrm{~K}$ (Shu 1977). In our model, it is the result of both higher densities and velocities produced by the fast compression wave propagating inwards. Fatuzzo et al. (2004) find that the mass infall rate indeed scales roughly linearily with the initial overdensity and the initial velocity field in their self-similar collapse solutions where the dense core is initially either overdense 


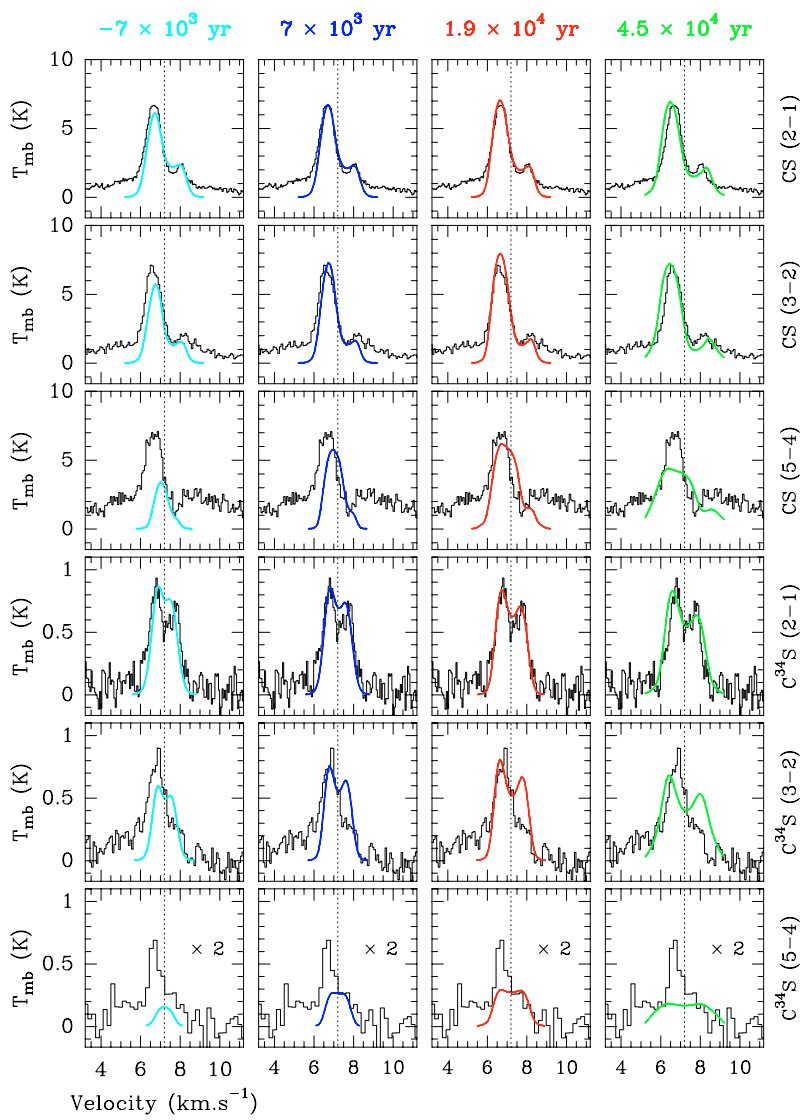

Fig. 10. $C S$ and $C^{34} S$ spectra in units of main-beam temperature observed toward the center of IRAS 4A (histograms). Each column shows a different model (thick line). Synthetic spectra corresponding to the collapse model described in Sect. 4.1 at times $-7,7,19$, and $45 \times 10^{3} \mathrm{yr}$ are superimposed from left to right (in light blue, blue, red, and green). For each model, the scaling of the abundance profile was adjusted to match the peak intensity of the $\mathrm{C}^{34} \mathrm{~S}(2-1)$ line. The dotted line indicates the systemic velocity of the envelope that was used to compute the radiative transfer (see Fig. 8).

compared to the singular isothermal sphere or has nonzero initial velocities.

In the framework of the self-similar inside-out collapse model with an effective sound speed including a non-thermal contribution, the "standard" accretion rate would be $\frac{\left(c_{\mathrm{s}}^{2}+\sigma_{\mathrm{NT}}^{2}\right)^{3 / 2}}{G}$ (Shu et al. 1987). This "effective" accretion rate would be at most 3 times higher than the pure isothermal one with the nonthermal velocity dispersion $\sigma_{\mathrm{NT}}=0.17 \mathrm{~km} \mathrm{~s}^{-1}$ derived for the inner parts of the envelope in Sect. 4.3, which is typical of the level of turbulence in prestellar condensations (e.g. Belloche et al. 2001), but probably even an upper limit for IRAS 4A since the smallest dispersion measured by Di Francesco et al. (2001) with PdBI is $0.09 \mathrm{~km} \mathrm{~s}^{-1}$. This cannot account for the high mass infall rate measured in the IRAS 4A envelope. On the other hand, the Larson-Penston similarity solution (Larson 1969; Penston 1969) has a uniform mass infall rate of $29 \times \frac{c_{s}^{3}}{G}$ at the point-mass formation, and its extension after the pointmass formation by Hunter (1977) has a mass infall rate approaching asymptotically $47 \times \frac{c_{\mathrm{s}}^{3}}{G}$. This value is closer to our result for IRAS $4 \mathrm{~A}$, which is not surprising since the LarsonPenston solution, among the numerous similarity solutions of isothermal gravitational collapse, can be physically interpreted as a model associated with a strong external compression wave
(Whitworth \& Summers 1985). Similarity solutions are helpful for understanding the physics of gravitational collapse, but they are not well suited to a detailed comparison with observations since they do not have realistic boundary conditions.

In contrast to the high mass infall rate measured in the IRAS 4A envelope, the spontaneous collapse of the very young Class 0 protostar IRAM 04191 in the Taurus molecular cloud is much less vigorous with a mass infall rate of only $2 \times \frac{c_{\mathrm{s}}^{3}}{G}$ (see Belloche et al. 2002). This could explain the order of magnitude difference between the bolometric luminosities of the two protostars that have approximately the same age since the beginning of the main accretion phase (see Lesaffre et al. 2005).

\subsection{Origin of the external compression}

The $\mathrm{N}_{2} \mathrm{H}^{+}(1-0)$ maps of Fig. 5 show that the material emitting at "blueshifted" velocities is displaced toward the South-West with respect to the material emitting at "redshifted" velocities and that both components have the same shell-like morphology at the edge of cavity 2 of Lefloch et al. (1998) seen in maps of continuum emission (see also Sandell \& Knee 2001; Hatchell et al. 2005). We interpret the velocity difference between the two components as the result of "inward" motions and conclude that the "blueshifted" material is in the background and moving toward the "redshifted" material along a direction projected onto the plane of the sky at PA $\sim 45^{\circ}$ in the IRAS 4 region and $\mathrm{PA} \sim 0^{\circ}$ in the SVS13/VLA 12 region. This geometry and this velocity structure remind us of an expanding shell, the center of which would be located in the background and southwest of NGC 1333. The "blueshifted" component would be the expanding shell itself while the "redshifted" component would be the ambient medium. In this scenario, the whole region around IRAS 4 and IRAS 2 should show signatures of "inward" motion, i.e. the motion of the "blueshifted" component toward the "redshifted" component. This might indeed be the case since Walsh et al. (2006) claim that they have detected "global infall" in this region. On smaller scales in the IRAS $4 \mathrm{~A}$ region, this scenario also fits, from a kinematical point of view, into the framework of the collapse model we investigated in Sect. 4. The external perturbation triggering the collapse of the dense core would then be the impact of this expanding shell on the ambient medium.

In this scenario, the origin of the external perturbation should be located in the background and southwest of the IRAS 4/SVS13 region. The perturbation could result from the expansion of an H II region, from a stellar wind, or from a protostellar outflow. A nearby bright star in the South-West is required for the first two assumptions. The star BD $+30^{\circ} 547$, with a spectral type between A 6 V and B 7 V (Aspin 2003, , although Cernis 1990 found a type G 2 IV), would be the best candidate. However, Preibisch (2003) finds with XMM-Newton that this star is in the foreground, so in our scenario, it cannot be the origin of the external perturbation in the IRAS 4 region. Since NGC 1333 is filled with many outflows (Knee \& Sandell 2000 ), the collapse of IRAS 4 could have been triggered by one of them. Like Hennebelle et al. (2003), Motoyama \& Yoshida (2003) numerically investigated the collapse of dense cores triggered by an external perturbation. They initiated the collapse by setting a nonzero inward velocity at the boundary of the dense core and found that the maximum value of the mass infall rate is proportional to the momentum given to the dense core by the external perturbation and, in particular, that an input momentum of $\sim 0.1 M_{\odot} \mathrm{km} \mathrm{s}^{-1}$ yields a mass infall rate of $\sim 1 \times 10^{-4} M_{\odot} \mathrm{yr}^{-1}$. The collapse of IRAS 4A could thus have been started by an 
external perturbation with a momentum of $\sim 0.1 M_{\odot} \mathrm{km} \mathrm{s}^{-1}$. This is a typical value for the present molecular outflows in NGC 1333 (Knee \& Sandell 2000). The shock created by a present or former outflow could therefore have triggered, directly or indirectly, the collapse of IRAS $4 \mathrm{~A}$. This would fit the scenario of shock-driven sequential star formation put forward by Warin et al. (1996) for NGC 1333. Indeed, the cavity in the South-West of IRAS 4A could be the relic of a former outflow (see Quillen et al. 2005). However, we have not yet found a good protostellar candidate that could have been the origin of such an outflow in the South-West.

We mentioned the limitation of our 1D spherical modeling for IRAS 4A in Sect. 4.3 and suggested an asymmetry of the source along the line of sight, the rear hemisphere being denser than the front hemisphere. This asymmetry could stem from the fast external compression itself, which is unlikely to be isotropic on the scale of the IRAS 4A envelope. Boss (1995) showed examples of such asymmetries in dense cores set in collapse by a shock wave hitting only one hemisphere (see his Fig. 2). It would be geometrically consistent with the idea that the compression wave that triggered the collapse of the IRAS 4A envelope is coming from the back, as deduced above from the morphology and kinematics of the $\mathrm{N}_{2} \mathrm{H}^{+}(1-0)$ maps.

\subsection{Non uniform turbulence}

We found in Sect. 4.3 that the non-thermal line broadening, which is likely to stem from turbulent motions, decreases toward the center of IRAS 4A from supersonic to subsonic velocities by nearly a factor of 3. A similar conclusion has already been drawn by Di Francesco et al. (2001) based on their $\mathrm{N}_{2} \mathrm{H}^{+}$interferometric data. The turbulence is thus supersonic in the outer parts of the IRAS 4A envelope but only subsonic in the inner dense region. The subsonic turbulence in the inner part of the envelope is reminiscent of the low level of turbulence found in prestellar condensations (e.g. in the $\rho$ Oph protocluster, see Belloche et al. 2001). It could then indicate the conditions prevailing in the dense core before the onset of collapse. In the outer parts of the envelope, the supersonic turbulence $\sigma_{\mathrm{NT}} \sim 0.50 \mathrm{~km} \mathrm{~s}^{-1}$, which we derived from the CS self-absorption, is nearly as large as the amplitude of the non-thermal motions deduced by Warin et al. (1996) from the low density tracer $\mathrm{C}^{18} \mathrm{O}(1-0)$ in the molecular cloud (see also Fig. 7 of Quillen et al. 2005, for $\mathrm{C}^{18} \mathrm{O}$ data with a better spatial resolution). The outer parts of the IRAS 4A envelope are therefore affected by the supersonic turbulence that permeates the whole cloud and that may have been powered by the numerous outflows located in NGC 1333 (Quillen et al. 2005). In the scenario we proposed in Sect. 5.2, the supersonic turbulence could have been generated in the outer parts of the IRAS 4A envelope by the expansion of the southwestern cavity.

\subsection{Rotation and binary formation}

Detecting rotation and measuring its magnitude in the envelope of IRAS 4A is not straightforward. We mentioned in Sect. 3.4 that the blue component of the low-optical-depth tracers shows a velocity gradient of $\sim 9.7 \mathrm{~km} \mathrm{~s}^{-1} \mathrm{pc}^{-1}$ at PA $\sim 38^{\circ}$ over $\sim 40^{\prime \prime}$, which is on the same order as the gradient measured in IRAM 04191 that we interpreted as rotation (Belloche et al. 2002). However, there are two caveats for IRAS 4A. First, the double-peaked structure of our single-dish low-opticaldepth spectra makes the analysis difficult in terms of rotation. Second, Di Francesco et al. (2001) observed $\mathrm{N}_{2} \mathrm{H}^{+}(1-0)$ with PdBI and measured centroid velocity differences of about $0.5-1 \mathrm{~km} \mathrm{~s}^{-1}$ over $\sim 12^{\prime \prime}$, which corresponds to a velocity gradient of $\sim 30-50 \mathrm{~km} \mathrm{~s}^{-1} \mathrm{pc}^{-1}$, at a position angle PA $\sim 135^{\circ}$. Their position angle was only a rough estimate and a fit to their centroid velocity map would give a position angle probably close to $90^{\circ}$ (see their Fig. 2b), but this is still $\sim 50^{\circ}$ away from our single-dish measurement so it is unclear whether these velocity gradients trace indeed rotation.

However, if we assume that the velocity gradient we measured in Sect. 3.4 is a good estimate of the amount of rotation in the envelope and suppose at first order that it is a solid-body rotation, then we find a ratio of rotation energy over gravitational energy of $\beta \sim 0.02$. This is similar to the conditions investigated by Hennebelle et al. (2004) when studying the fragmentation process in a rotating core set in collapse by a fast external compression. Their main conclusion was that a fast compression promotes fragmentation and the formation of multiple protostars. With a very rapid compression $(\phi=0.1)$, they show that this process occurs through the formation of a ring which becomes unstable very quickly. It breaks up into several pieces that, in their example, merge into two protostars. For their simulation with a dense core of $1 M_{\odot}$, the proto-binary separation is about $140 \mathrm{AU}$ (see their Fig. 7), which corresponds to $840 \mathrm{AU}$ if we normalize their model to the mass of the IRAS 4A envelope $\left(6 M_{\odot}\right)$. IRAS $4 \mathrm{~A}$ is indeed a binary system with a separation of $1.8^{\prime \prime}$, i.e. $570 \mathrm{AU}$ at a distance of $318 \mathrm{pc}$ (see Looney et al. 2000; Reipurth et al. 2002). It is therefore tempting to conclude that the formation of the IRAS 4A binary system results from the fast external compression that triggered the collapse of the dense core.

\subsection{Collapse of NGC1333 IRAS 4B}

As for IRAS 4A, Di Francesco et al. (2001) find an inverse P-Cygni profile toward the Class 0 protostar IRAS 4B (Sandell et al. 1991). They conclude that it is also collapsing, with a similar mass infall rate of $3.7 \times 10^{-5} M_{\odot} \mathrm{yr}^{-1}$. Since it lies along the same $\mathrm{N}_{2} \mathrm{H}^{+}$filament as IRAS 4A (see Fig. 5) and is in a similar evolutionary stage, we suggest that the gravitational collapse of its envelope was triggered by the same external perturbation, the expanding shell suggested in Sect. 5.2.

We note that IRAS 4B is an $11^{\prime \prime}$ binary and possibly a system with even higher multiplicity (see Lay et al. 1995; Looney et al. 2000). However, the main companion was not detected in continuum emission at $3.6 \mathrm{~cm}$ by Reipurth et al. (2002) and is probably much more evolved than the primary (Looney et al. 2000). This raises the question of whether it really belongs to the IRAS 4B system or is physically unrelated and appears close to the Class 0 protostar in the plane of the sky just by chance.

\subsection{Alternative interpretations?}

In the past, it has been claimed that the NGC 1333 molecular cloud includes several independent velocity components seen in emission and/or absorption (see Langer et al. 1996; Choi 2001; Choi et al. 2004). In particular, Choi et al. (2004) reject the infall interpretation of Di Francesco et al. (2001) and instead propose a foreground absorbing layer at $8 \mathrm{~km} \mathrm{~s}^{-1}$ that is physically unrelated to IRAS 4 A. They assume a very low systemic velocity of $6.7 \mathrm{~km} \mathrm{~s}^{-1}$ for IRAS 4A, yielding highly redshifted absorption dips in the optically thick spectra, and argue that standard collapse models like the inside-out collapse model (Shu 1977) would never produce such highly redshifted dips given the age 
of the protostar, and that the faster Larson-Penston model would produce linewidths that are much too broad to fit these highly redshifted dips. However, the model presented in Sect. 4 shows that a systemic velocity of $7.2 \mathrm{~km} \mathrm{~s}^{-1}$ and a collapse induced by a fast external compression can fit both the optically thick and thin lines reasonably well. The fit to our data set does not require any additional absorbing layer that is physically unrelated to IRAS 4A, as claimed by Choi et al. (2004). Our scenario, which requires only one source, is simpler. Therefore we think it is more likely.

\section{Summary and conclusions}

We have carried out a detailed analysis of the physical structure of the envelope of the Class 0 protostar IRAS $4 \mathrm{~A}$ in the NGC 1333 molecular cloud. Our main results and conclusions are as follows:

1. The density profile deduced from previous interferometric and single-dish millimeter continuum measurements is steep $(p=2.3)$, and the envelope is much denser than a singular isothermal sphere at $10 \mathrm{~K}$.

2. Our new set of molecular line data obtained with the IRAM 30 m telescope shows the classical spectroscopic signature of collapse with absorption dips of optically thick lines redshifted by $0.5 \mathrm{~km} \mathrm{~s}^{-1}$ with respect to the systemic velocity of the source traced by optically thin lines.

3 . Both the density structure deduced from the continuum observations and our set of $\mathrm{CS}, \mathrm{C}^{34} \mathrm{~S}$, and $\mathrm{N}_{2} \mathrm{H}^{+}$spectra can be well fitted by a model of collapse induced by a fast external compression with a time of $1-2 \times 10^{4} \mathrm{yr}$ after the formation of the central protostar. This age is consistent with the lifetime estimated for Class 0 protostars.

4. We deduce a very high mass infall rate of $0.7-2 \times$ $10^{-4} M_{\odot} \mathrm{yr}^{-1}$ in the envelope of IRAS 4A. It results from both the overdensity and the high velocities generated by the fast external compression.

5. Our radiative transfer modeling reveals a strong decrease in the level of turbulence from supersonic velocities in the outer regions to subsonic velocities near the center of the IRAS 4A envelope. The subsonic turbulence is probably a relic of the conditions prevailing in the dense core before the onset of collapse.

6. We found indications of the presence of an expanding shell in the vicinity of IRAS 4A, which could be the origin of the compression. This expanding shell could have simultaneously triggered the collapse of the nearby Class 0 protostar IRAS 4B. However, we did not find any evidence of a nearby bright star that could have initiated this compression through the expansion of an $\mathrm{H}$ II region or through a strong wind. On the other hand, the collapse of IRAS 4A and IRAS 4B could have been triggered, directly or indirectly, by an outflow, but we have not found a good protostellar candidate yet.

Acknowledgements. We would like to thank Jennifer Hatchell for her help with the software SPECX and Holger Müller for the new $\mathrm{HCO}^{+}$entry in the CDMS database. We also thank the referee, Mario Tafalla, for helpful comments.

\section{References}

André, P., Ward-Thompson, D., \& Barsony, M. 1993, ApJ, 406, 122 André, P., Ward-Thompson,D., \& Barsony, M. 2000, in Protostars and planets IV, ed. V. Mannings, A. Boss, \& S. Russell (Tucson: Univ. Arizona Press), 59 André, P., Belloche, A., Hennebelle, P., \& Ward-Thompson, D. 2004, Baltic Astronomy, 13, 392
Aspin, C. 2003, AJ, 125, 1480

Belloche, A., \& André, P. 2004, A\&A, 419, L35

Belloche, A., André, P., \& Motte, F. 2001, From Darkness to Light: Origin and Evolution of Young Stellar Clusters, ASP Conf. Ser., 243, 313

Belloche, A., André, P., Despois, D., \& Blinder, S. 2002, A\&A, 393, 927

Blake, G. A., Sandell, G., van Dishoeck, E. F., et al. 1995, ApJ, 441, 689

Blinder, S. 1997, Thesis, Université de Bordeaux I

Bonnor, W. B. 1956, MNRAS, 116, 351

Boss, A. P. 1995, ApJ, 439, 224

Buisson, G., Desbats, L., Duvert, G., et al. 2002, CLASS manual, version 4.0 (IRAM)

Caselli, P., \& Dore, L. 2005, A\&A, 433, 1145

Caselli, P., Walmsley, C. M., Zucconi, A., et al. 2002, ApJ, 565, 331

Cernis, K. 1990, Ap\&SS, 166, 315

Choi, M. 2001, ApJ, 553, 219

Choi, M. 2005, ApJ, 630, 976

Choi, M., Kamazaki, T., Tatematsu, K., \& Panis, J.-F. 2004, ApJ, 617, 1157

de Zeeuw, P. T., Hoogerwerf, R., de Bruijne, J. H. J., Brown, A. G. A., \& Blaauw, A. $1999, \mathrm{AJ}, 117,354$

Di Francesco, J., Myers, P. C., Wilner, D. J., Ohashi, N., \& Mardones, D. 2001, ApJ, 562, 770

Dore, L., Caselli, P., Beninati, S., et al. 2004, A\&A, 413, 1177

Evans, N. J. II 1999, ARA\&A, 37, 311

Fatuzzo, M., Adams, F. C., \& Myers, P. C. 2004, ApJ, 615, 813

Flower, D. R. 1999, MNRAS, 305, 651

Getman, K. V., Feigelson, E. D., Townsley, L., et al. 2002, ApJ, 575, 354

Gottlieb, C. A., Myers, P. C., \& Thaddeus, P. 2003, ApJ, 588, 655

Gregersen, E. M., Evans, N. J., Zhou, S., \& Choi, M. 1997, ApJ, 484, 256

Hatchell, J., Richer, J. S., Fuller, G. A., et al. 2005, A\&A, 440, 151

Hennebelle, P., Whitworth, A. P., Gladwin, P. P., \& André, P. 2003, MNRAS, 340,870

Hennebelle, P., Whitworth, A. P., Cha, S.-H., \& Goodwin, S. P. 2004, MNRAS, 348,687

Hildebrand, R. H. 1983, QJRAS, 24, 267

Hunter, C. 1977, ApJ, 218, 834

Knee, L. B. G., \& Sandell, G. 2000, A\&A, 361, 671

Lada, C. J., \& Lada, E. A. 2003, ARA\&A, 41, 57

Lada, C. J., Alves, J., \& Lada, E. A. 1996, AJ, 111, 1964

Langer, W. D., Castets, A., \& Lefloch, B. 1996, ApJ, 471, L111

Larson, R. B. 1969, MNRAS, 145, 271

Lay, O. P., Carlstrom, J. E., \& Hills, R. E. 1995, ApJ, 452, L73

Lefloch, B., Castets, A., Cernicharo, J., Langer, W. D., \& Zylka, R. 1998, A\&A, 334, 269

Lesaffre, P., Belloche, A., Chièze, J.-P., \& André, P. 2005, A\&A, 443, 961

Looney, L. W., Mundy, L. G., \& Welch, W. J. 2000, ApJ, 529, 477

Looney, L. W., Mundy, L. G., \& Welch, W. J. 2003, ApJ, 592, 255

Mardones, D., Myers, P. C., Tafalla, M., et al. 1997, ApJ, 489, 719

Maret, S., Ceccarelli, C., Caux, E., et al. 2004, A\&A, 416, 577

Matthews, H., \& Jenness, T. 1997, Specx Cookbook Reduction of millimetre wave data, Starlink Document sc8

Motoyama, K., \& Yoshida, T. 2003, MNRAS, 344, 461

Motte, F., \& André, P. 2001, A\&A, 365, 440

Müller, H. S. P., Schlöder, F., Stutzki, J., \& Winnewisser, G. 2005, J. Mol. Struct., 742,215

Myers, P. C., Evans, N. J. II, \& Ohashi, N. 2000, in Protostars and planets IV, ed.

V. Mannings, A. Boss, \& S. Russell (Tucson: Univ. Arizona Press), 217

Onishi, T., Mizuno, A., \& Fukui, Y. 1999, PASJ, 51, 257

Padman, R. 1993, SPECX Users' Manual, Version 6.3

Penston, M. V. 1969, MNRAS, 144, 425

Preibisch, T. 2003, A\&A, 401, 543

Quillen, A. C., Thorndike, S. L., Cunningham, A., et al. 2005, ApJ, 632, 941

Reipurth, B., Rodríguez, L. F., Anglada, G., \& Bally, J. 2002, AJ, 124, 1045

Sandell, G., \& Knee, L. B. G. 2001, ApJ, 546, L49

Sandell, G., Aspin, C., Duncan, W. D., Russell, A. P. G., \& Robson, E. I. 1991, ApJ, 376, L17

Schmid-Burgk, J., Muders, D., Müller, H. S. P., \& Brupbacher-Gatehouse, B. 2004, A\&A, 419, 949

Shu, F. H. 1977, ApJ, 214, 488

Shu, F. H., Adams, F. C., \& Lizano, S. 1987, ARA\&A, 25, 23

Smith, K. W., Bonnell, I. A., Emerson, J. P., \& Jenness, T. 2000, MNRAS, 319, 991

Tafalla, M., Mardones, D., Myers, P. C., et al. 1998, ApJ, 504, 900

Terebey, S., Chandler, C. J., \& André, P. 1993, ApJ, 414, 759

Walsh, A. J., Bourke, T. L., \& Myers, P. C. 2006, ApJ, 637, 860

Warin, S., Castets, A., Langer, W. D., Wilson, R. W., \& Pagani, L. 1996, A\&A, 306, 935

Whitworth, A., \& Summers, D. 1985, MNRAS, 214, 